\title{
Can Remote Patient Management Improve Outcomes in Peritoneal Dialysis?
}

\author{
Longkai Li ${ }^{a} \cdot$ Jeffrey Perl ${ }^{\mathrm{b}, \mathrm{c}}$
}

a Division of Nephrology, First Affiliated Hospital of Dalian Medical University, Dalian, China; ${ }^{b}$ Division of Nephrology, St. Michael's Hospital, Toronto, ON, Canada; 'Keenan Research Center in the Li Ka Shing Knowledge Institute, St. Michael's Hospital, Toronto, ON, Canada

\begin{abstract}
Remote patient management (RPM) for patients receiving peritoneal dialysis (PD) is an emerging technology whereby a patients' biometric information (including weight, blood pressure, and other parameters) can be remotely monitored by providers along with important PD treatment information. As part of an evolving field of telemedicine, RPM has the potential to improve clinical outcomes for patients receiving PD, reduce resource utilization, and improve PD uptake, and positively impact a patients' experience with PD. Preliminary reports of RPM are encouraging, however, research in the field is limited to small, single center studies, and remains largely observational; interventions have been heterogenous in terms of what clinical and treatment parameters are being remotely monitored and what outcomes are under evaluation. In the present review, we will describe the evolving field of RPM in PD, discuss potential benefits of the technology while highlighting existing and important future areas for research.

() 2019 S. Karger AG, Basel
\end{abstract}

\section{Introduction}

Remote patient management (RPM) uses digital technologies to collect health data from individuals in 1 location, such as a patient's home, and electronically transmit the information to healthcare providers in a different location for as- 
sessment and recommendation $[1,2]$. Telehealth remains a broader definition, and the definition according to the World Health Organization involves the use of telecommunications and virtual technology to deliver healthcare outside of traditional healthcare facilities [3]. The overarching goal of RPM and telemedicine is to improve patients' health status, which may be by expediting diagnosis, and/or facilitating prompt treatment, disease prevention [4]. The ultimate goal would be to reduce adverse events (i.e., hospitalization), improve survival, and patient reported outcomes such as treatment satisfaction and quality of life while reducing healthcare resources and costs.

The use of RPM has been successfully applied across the management of other chronic diseases including congestive heart failure, diabetes mellitus, hypertension, and chronic obstructive pulmonary disease [5]. In chronic kidney disease patients particularly, the use of wireless home blood pressure monitoring and smart phone applications have been useful in allowing patients with uncontrolled hypertension to gain better control of blood pressure [6]. RPM is especially germane to home dialysis patients, who may live far from the dialysis facility, and who perform their treatments autonomously independent of the clinic and often using complex medical devices. RPM may then extend the clinical encounter outside of the facility to the patient's home by employing the use of wireless technology to monitor clinical and dialysis treatment parameters and potentially facilitate more prompt changes in the dialysis prescription [7]. In the United States, RPM in home dialysis is likely to expand and complement recent legislation that states that individuals receiving home dialysis therapy may choose to receive monthly end-stage kidney disease (ESKD)-related clinical assessments furnished on or after January 1, 2019 via telehealth [8]. Therefore, in the present chapter, we will highlight key considerations in evaluating the potential benefits of RPM for the care of patients receiving peritoneal dialysis (PD). We will describe evolving research regarding the use of RPM among home dialysis patients. Lastly, we will identify important areas for future research.

\section{Can RPM Improve PD Utilization?}

Among high income countries in the last several years, there has been significant decline in PD utilization [9]. In an era of improving outcomes for patients treated with home-based PD, policy-makers and healthcare providers continue to focus on increasing incident PD utilization as an attractive cost-containment strategy [10]. Given that, the fear of autonomously performing home dialysis remains one of the barriers which may limit wider uptake of home dialysis [11, 


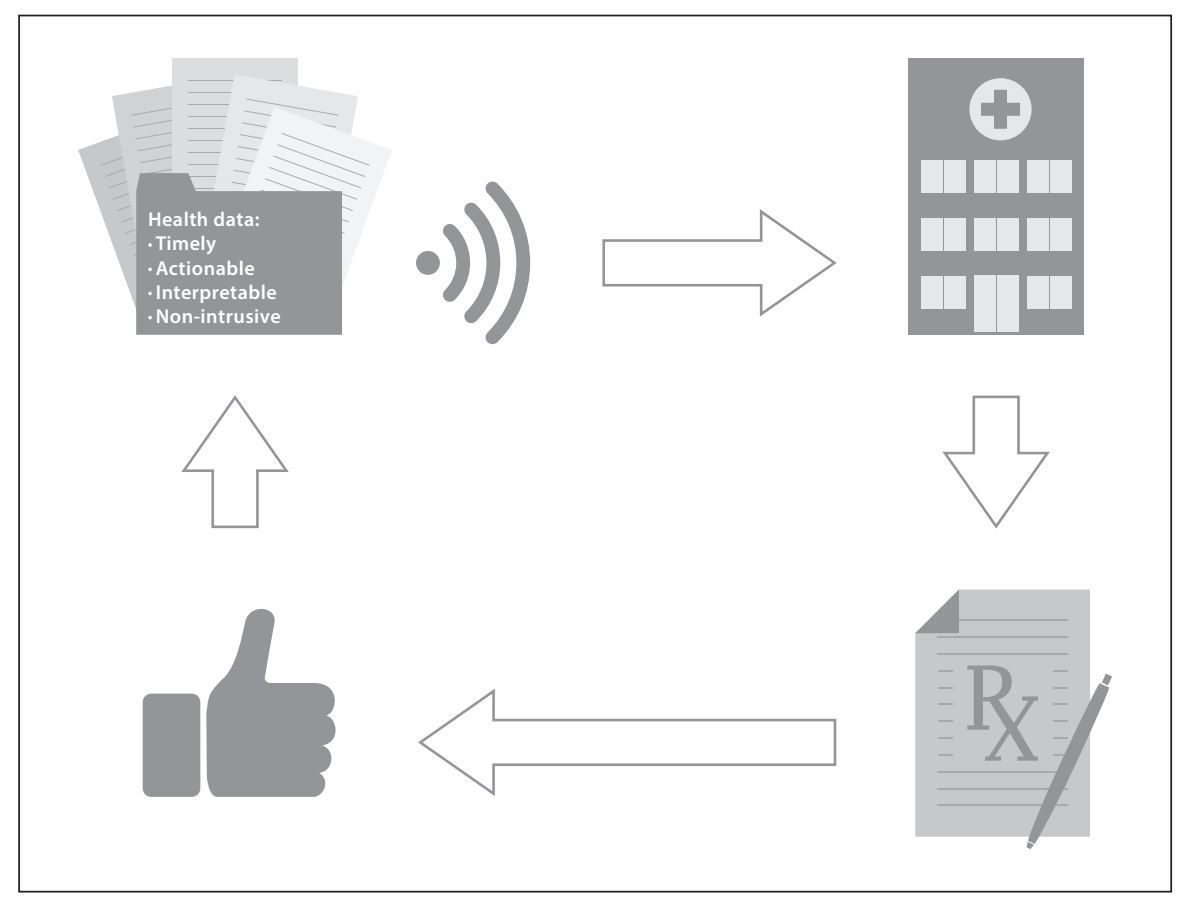

Fig. 1. The "loop" of RPM. Health data that are transmitted to the clinic should be timely, actionable, interpretable and non-intrusive, and should be addressed by the clinic with a change in the treatment plan (i.e., dialysis prescription, medication change, counselling) that yields a positive health outcome.

12]. RPM may help overcome this barrier by giving patients more confidence to perform dialysis treatments at home. Furthermore, limiting clinic visits by the use of RPM as an adjunct strategy to a telemedicine platform may further increase the utilization of PD as an attractive option for many patients. Lastly, among long-term care facilities who may have been previously concerned about the responsibility of performing PD treatments, RPM may provide these clinics more support to be able to consider providing PD for their residents.

\section{RPM in PD: How Are We Monitoring? What Are We Monitoring?}

For RPM to be successful in improving outcomes for patients and care partners, relevant health data must be transmitted to the clinic efficiently, interpreted correctly, and a treatment change initiated that leads to a positive health outcome (Fig. 1). Any breakdown in this process in the "loop of RPM care" may attenuate the potential benefits of RPM. 


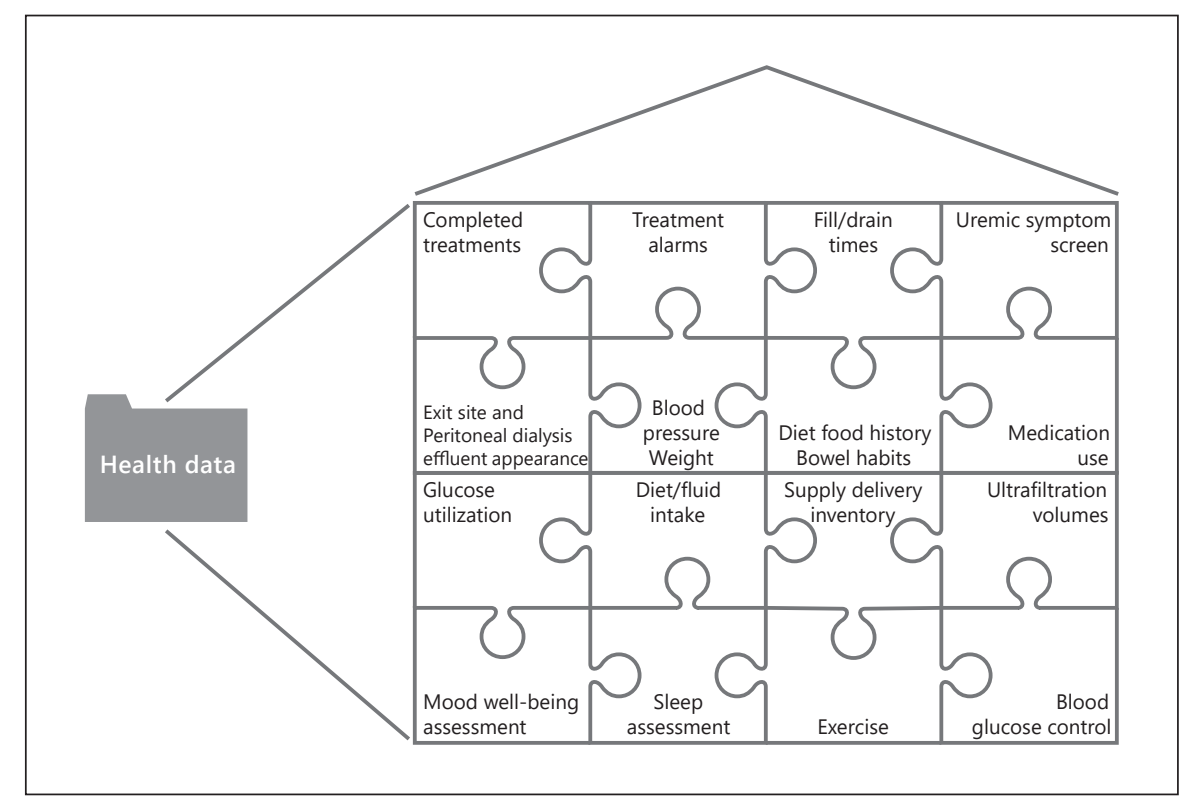

Fig. 2. Possible health data amenable to remote monitoring.

In addition to monitoring of biometric information including weight, pulse, blood pressure, blood sugar levels, RPM in PD also offers the opportunity to monitor many aspects of the PD prescription. To date, most RPM has focused on aspects of automated PD (APD) treatments as opposed to continuous ambulatory PD (CAPD) treatments. During overnight treatment, the clinic has the opportunity to monitor treatment adherence, in some cases the selection of PD fluids and monitor the speed and fluidity of overnight APD exchanges and final total overnight peritoneal ultrafiltration (UF). Figure 2 shows a list of potential aspects of RPM that could be theoretically monitored. If too much information is presented to the clinician and treatment team, then the concern is that important treatment information may be diluted and obscured, and there is a possibility of receiving more information than can be reasonably processed. Taken together, it is important to have an evidence-based and rationale approach to what information is monitored and with what frequency. It is also possible that certain aspects of PD therapy may be more germane at different timepoints during a patient's therapy and the spectrum of what is monitored, and the level of detail may need to change over time. For example, monitoring of peritoneal UF may be particularly important among anuric patients where among this group a minimum daily peritoneal UF volume is an important predictor of survival $[13,14]$. In the following sections, we will discuss what aspects of RPM have been examined and how they relate to patient outcomes on PD. 


\section{PD Access Dysfunction}

$\mathrm{PD}$ catheter malfunction is a common cause of early PD attrition [15]. It may be more important to be attentive to RPM parameters which may relate to PD catheter function, particularly early in the course of PD therapy. For example, prolonged drain times as identified via RPM may identify problems with PD catheter outflow obstruction, which may lead to treatment alarms and/or drain pain which may cause patient discomfort and/or frustration early on in the therapy. Early identification of these problems may allow for a change in PD prescription to remediate these issues (i.e., fewer APD cycles, use of tidal therapy) and/or instructions for more vigorous use of laxatives and in some cases prompter PD catheter revision. Dreppe et al. [16] reported one APD patient to have prolonged drain times via alerts received via RPM. The patient was called for urgent clinical evaluation and radiological investigations with a diagnosis of catheter displacement. After surgical repositioning of the catheter, drain volumes and drain times were again normal [16]. The availability and application of the RPM platform to monitor and modify APD treatment parameters may offer clinicians the potential for earlier identification of PD catheter dysfunction. Whether or not more prompt identification and intervention translates into improved patient retention on $\mathrm{PD}$ remains to be evaluated in future research.

\section{Treatment Adherence}

Non-adherence to PD treatments, defined as performing less than $90 \%$ of prescribed PD exchanges, was found to be as high as 30\% of patients in the first 6 months of PD therapy in a single center study [17] and prevalent in over one third of patients in another multicenter cohort study [18]. In a recent metaanalysis, non-adherence varied across studies: $2.6-53 \%$ for dialysis exchanges, $3.9-85 \%$ for medication, and $14.4-67 \%$ for diet/fluid restrictions [19]. In the single center study, non-adherent patients had higher rates of death, transfer to hemodialysis (HD) due to uremia, and hospitalization episodes compared to those performing their PD therapy [17]. The use of RPM can rapidly identify consistent non-adherence to PD therapy and is a first step in informing interventions to promote treatment adherence among these patients which may translate into improved patient outcomes. 


\section{Hospitalization and Emergency Department Visit Reduction}

Patients with ESKD experience high rates of hospitalization and emergency department (ED) visits and disproportionate readmission rates compared to the general population [20-22]. Readmission rates may be higher among PD patients compared to those receiving in-center HD in part which may relate to differences in the frequency of contact with the dialysis care team between patients receiving facility-based $\mathrm{HD}$ vs. $\mathrm{PD}$ [23]. It is tempting to speculate that $\mathrm{RPM}$ may be one tool to mitigate hospitalization and readmission risks. Among highrisk facility-based HD patients a 3-year study of 99 patients, submission of biometric information (weight, blood pressure, oxygen saturation, blood pressure) led to lower hospitalization, hospitalization days, and hospital and ED costs among 43 patients compared to 56 patients receiving usual care [24]. In a simulation study in Japan reported by Uchiyama et al. [25], the use of RPM among 12 patient scenarios was shown to reduce total healthcare consumption, particularly in domains unplanned hospital visits and emergency room visits. Moreover, Lew et al. [26] demonstrated that daily remote biometric monitoring of blood pressure and weight among 269 patients receiving PD was associated with lower outpatient visit claims for those aged 18-54 years (not in the overall cohort) and that overall costs were lower if remote monitoring included blood pressure and weight. Use of remote monitored collected blood pressure was associated with increased days of hospitalization and higher odds of hospitalization. These conflicting results may relate to the notion that in certain instances, RPM may identify problems at an earlier stage thereby minimizing the need for hospital admission by addressing these at an earlier stage. On the contrary, having more biometric information at hand may also lead to more interventions and potentiate additional inpatient visits. Further multi-center research is needed to better assess the impact of RPM on rates of hospitalization and ED visits.

\section{PD-Related Infections}

Through both remote monitoring and 2-way video-teleconferencing capability, the care team can reach a clear diagnosis whether there might be signs of infection when patients send high-resolution pictures or video of their PD catheter exit site or drain bags for evaluation [5]. In a study by Nayak et al. [27], a retrospective analysis of 246 patients in India demonstrated results using an internetbased RPM system that allowed both exit site and peritoneal effluent images to be uploaded by patients and transmitted to the clinic. While these technologies likely aid in the prompt treatment of PD-related infections including exit site 
infections and peritonitis which may improve outcomes after infection, to date peritonitis and exit site prevention strategies directly targeted via RPM are lacking and future innovation is needed in this regard.

\section{Patient-Reported Outcomes}

Patients receiving PD, in general, rate their care higher than those receiving HD which likely reflects the empowerment and support provided by the treatment team to facilitate patients as active participants in their own care [28]. In general, the study by Lew et al. [29] demonstrated that in a high-income country such as the United States most of their patients had the technology (owning a computer and cell phone, and knowing how to use them) and the interest for participating in RM. It is conceivable that the use of RPM by patients may improve particular domains of patient reported outcomes including therapy confidence, treatment satisfaction, and improved self-efficacy, and may also have the potential to negatively impact domains of illness intrusiveness and disease burden. Initial reports suggest that patients using RPM are better able to identify changes about their health status, and experienced enhanced accountability, self-efficacy, and motivation to make health behavior changes [30]. A study by Cao et al., employing instant messaging software among 160 PD patients in China, showed that among the 80 patients in the intervention group a significantly higher reported degree of satisfaction compared to those in the non-intervention group [31]. In a project evaluating the impact of biometric monitoring of PD patient physiologic parameters (i.e., weight, blood pressure) by Magnus et al. [32], survey results among the 300 participants revealed that over 80 percent indicated that they were satisfied or completely satisfied with the system. Taken together, preliminary reports suggest that RPM may have the capability to increase patient satisfaction, however, it is likely that these results may vary by the type and invasiveness of the remote monitoring technology employed. As a result, all future studies of RPM technology among PD patients should include both qualitative and quantitative evaluation of patient reported outcomes and patient-perceived benefits. Such studies are currently underway [33, 34].

\section{RPM: Lessons Learnt from Home Hemodialysis}

Compared to $\mathrm{PD}$, home hemodialysis (HHD) patients face increased technical aspects of performing home dialysis treatments and a potentially greater risk of significant intradialytic hemodynamic changes and access-related serious ad- 
verse events. As a result, it is possible that the utility of RPM may be that much greater among HHD patients compared to those receiving PD. Among patients receiving conventional $\mathrm{HD}$ contemplating conversion to $\mathrm{HHD}$ in one study, RPM was seen as an expectation particularly in the transition phase but is unlikely to be the sole factor impacting a patient's attitude to consider HHD [35]. In a recent evaluation of a remote monitoring platform for HHD patients in Australia, qualitative data showed that the HHD system was able to improve patients' feelings of being connected with their healthcare professionals and thus enhance their self-care on HHD [36].

RPM parameters in HHD would include not only the monitoring of biometric parameters, but also HD machine parameters, alarms and the responsiveness of the patients and/or care partner to them. Initial experience in the nocturnal HHD program in Toronto mandated RPM among all patients. The system employed software that allowed for live remote monitoring of all functions of the HD machine with an observer trained by the group observing patients remotely. During the time of its widespread operation, the number of alarms decreased by 1.7 per night to 0.1 per night [37]. Furthermore, serious adverse events were unlikely to be prevented by the use of RPM and were likely exceedingly rare among HHD patients. These events largely relate to air emboli and vascular access dislodgement events $[37,38]$. As a result, RPM has been increasingly abandoned across HHD programs in Canada and remains optional across many programs. Further study is needed to better understand the role of RPM in the care of HHD patients.

\section{Future Evaluation of RPM}

The use of RPM in the care of PD patients represents a promising and powerful resource. It is likely that this technology will be an important adjunct in shaping the expansion of telemedicine and "telenephrology" to an already increasingly frail and elderly ESKD patient population where travel to and from the dialysis clinic is already challenging.

Research on which aspects of RPM should be monitored, in what patient population and over what time course of PD therapy is desperately needed. Furthermore, there is a need for the evaluation of well-defined algorithms that clinicians and providers can easily apply to address common troubleshooting scenarios identified by RPM. Moreover, important outcomes need to be addressed via randomized controlled trials and by leveraging the infrastructure of multicenter observational studies [39]. The recently published Standardized Outcomes in Nephrology-PD study which aimed to establish a core outcome set for 
trials in patients receiving $\mathrm{PD}$, identified $\mathrm{PD}$ technique survival, $\mathrm{PD}$-related infection, and life participation activities as the top priorities for PD research outcomes among a stakeholder group comprised of patients, providers, and healthcare professionals [40]. To date, evidence generation in RPM has been limited in these areas. In Canada, the CONNECT trial led by Jeffs et al. [41] seeks to randomize 500 patients to an eQ Connect ${ }^{\mathrm{TM}}$ platform telehome monitoring device (a mobile tablet) to support them with their PD (communication, treatment tracking, supply tracking, appointment reminders, educational content) (trial registration: NCT02670512) [30]. The primary outcome is a composite of technique failure (switching to HD for $\geq 12$ weeks), infections (peritonitis, exit-site, tunnel), and hospital encounters (ER visits, hospitalizations). To date, 340 patients have been enrolled (personal communication, A Jain) and the PD community will be anxiously awaiting the important study findings.

Lastly, RPM is somewhat of an unfortunate term because it implies that there is a unidirectional flow of health information from the patient to the clinic. However, it is a platform designed to better enhance bidirectional communication of information between patients and providers. To maximize its effectiveness, patients should also be encouraged to be custodians over their health information such that RPM can maximally improve the overall self-efficacy, disease management, and awareness for patients receiving PD.

\section{Disclosure Statement}

L.L. and J.P. received speaking honoraria from Baxter Healthcare, Fresenius Medical Care, Dialysis Clinic Incorporated, Davita Healthcare Partners, and consulting fees from Baxter Healthcare, Davita Healthcare Partners as well as research support from Arbor Research Collaborative for Health.

\section{References}

1 Vegesna A, Tran M, Angelaccio M, Arcona S: Remote patient monitoring via non-invasive digital technologies: a systematic review. Telemed J E Health 2017;23:3-17.

2 http://telehealth.med.miami.edu/what-is-telehealth.

3 https://www.who.int/sustainable-development/ health-sector/strategies/telehealth/en/.

4 Rohatgi R, Ross MJ, Majoni SW: Telenephrology: current perspectives and future directions. Kidney Int 2017;92:1328.
5 Rosner MH, Lew S, Conway P, Ehrlich J, Jarrin R, Patel UD, Rheuban K, Robey RB, Sikka N, Wallace E: Perspectives from the kidney health initiative on advancing technologies to facilitate remote monitoring of patient self-care in RRT. Clin J Am Soc Nephrol 2017;12:1900-1909.

6 Ong SW, Jassal SV, Miller JA, Porter EC, Cafazzo JA, Seto E, Thorpe KE, Logan AG: Integrating a smartphone-based self-management system into usual care of advanced CKD. Clin J Am Soc Nephrol 2016;11:1054-1062. 
7 Wallace EL, Rosner MH, Alscher MD, Schmitt CP, Jain A, Tentori F, Firanek C, Rheuban KS, Florez-Arango J, Jha V: Remote patient management for home dialysis patients. Kidney Int Reports 2017;2:1009-1017.

8 Bieber SD, Weiner DE: Telehealth and home dialysis a new option for patients in the united states. Clin J Am Soc Nephrol 2018;13:12881290.

9 Jain AK, Blake P, Cordy P, Garg AX: Global trends in rates of peritoneal dialysis. J Am Soc Nephrol 2012;23:533-544.

10 Perl J, Wald R, Bargman JM, Na Y, Jassal SV, Jain AK, Moist L, Nessim SJ: Changes in patient and technique survival over time among incident peritoneal dialysis patients in Canada. Clin J Am Soc Nephrol 2012;7:1145-1154.

11 Pipkin M, Eggers PW, Larive B, Rocco MV, Stokes JB, Suri RS, Lockridge RS Jr; Frequent Hemodialysis Network Trial Group: Recruitment and training for home hemodialysis: experience and lessons from the Nocturnal Dialysis Trial. Clin J Am Soc Nephrol 2010;5:1614-1620.

12 Cafazzo JA, Leonard K, Easty AC, Rossos PG, Chan CT: Patient-perceived barriers to the adoption of nocturnal home hemodialysis. Clin J Am Soc Nephrol 2009;4:784-789.

13 Brown EA, Davies SJ, Rutherford P, Meeus F, Borras M, Riegel W, Divino Filho JC, Vonesh E, van Bree M: Survival of functionally anuric patients on automated peritoneal dialysis: the European APD Outcome Study. J Am Soc Nephrol 2003; 14:2948-2957.

14 Lin X, Lin A, Ni Z, Yao Q, Zhang W, Yan Y, Fang W, Gu A, Axelsson J, Qian J: Daily peritoneal ultrafiltration predicts patient and technique survival in anuric peritoneal dialysis patients. Nephrol Dial Transplant 2010;25:2322-2327.

15 Kolesnyk I, Dekker FW, Boeschoten EW, Krediet RT: Time-dependent reasons for peritoneal dialysis technique failure and mortality. Perit Dial Int 2010;30:170-177.

16 Drepper VJ, Martin PY, Chopard CS, Sloand JA: Remote patient management in automated peritoneal dialysis: a promising new tool. Perit Dial Int 2018;38:76-78.

17 Bernardini J, Nagy M, Piraino B: Pattern of noncompliance with dialysis exchanges in peritoneal dialysis patients. Am J Kidney Dis 2000;35:11041110.

18 Kutner NG, Zhang R, McClellan WM, Cole SA: Psychosocial predictors of non-compliance in haemodialysis and peritoneal dialysis patients. Nephrol Dial Transplant 2002;17:93-99.

19 Griva K, Lai AY, Lim HA, Yu Z, Foo MW, Newman SP: Non-adherence in patients on peritoneal dialysis: a systematic review. PLoS One 2014; 9:e89001.
20 Molnar AO, Moist L, Klarenbach S, Lafrance JP, Kim SJ, Tennankore K, Perl J, Kappel J, Terner M, Gill J, Sood MM: Hospitalizations in dialysis patients in canada: a national cohort study. Can J Kidney Health Dis 2018;5:2054358118780372.

21 Chan L, Chauhan K, Poojary P, Saha A, Hammer E, Vassalotti JA, Jubelt L, Ferket B, Coca SG, Nadkarni GN: National estimates of 30-day unplanned readmissions of patients on maintenance hemodialysis. Clin J Am Soc Nephrol 2017; 12:1652-1662.

22 Harel Z, Wald R, McArthur E, Chertow GM, Harel S, Gruneir A, Fischer HD, Garg AX, Perl J, Nash DM, Silver S, Bell CM: Rehospitalizations and emergency department visits after hospital discharge in patients receiving maintenance hemodialysis. J Am Soc Nephrol 2015;26:31413150.

23 Misra PS, Bargman JM, Perl J: Survival comparisons in home dialysis: where you finish depends on where you start. Am J Kidney Dis 2016;67: 13-15.

24 Minatodani DE, Berman SJ: Home telehealth in high-risk dialysis patients: a 3-year study. Telemed J E Health 2013;19:520-522.

25 Uchiyama K, Washida N, Yube N, Kasai T, Shinozuka K, Morimoto K, Hishikawa A, Inoue H, Urai H, Hagiwara A, Fujii K, Wakino S, Deenitchina S, Itoh $\mathrm{H}$ : The impact of a remote monitoring system of healthcare resource consumption in patients on automated peritoneal dialysis (APD): a simulation study. Clin Nephrol 2018;90:334-340.

26 Lew SQ, Sikka N, Thompson C, Magnus M: Impact of remote biometric monitoring on cost and hospitalization outcomes in peritoneal dialysis. J Telemed Telecare 2018:1357633X18784417.

27 Nayak KS, Ronco C, Karopadi AN, Rosner MH: Telemedicine and remote monitoring: supporting the patient on peritoneal dialysis. Perit Dial Int 2016;36:362-366.

28 Rubin HR, Fink NE, Plantinga LC, Sadler JH, Kliger AS, Powe NR: Patient ratings of dialysis care with peritoneal dialysis vs hemodialysis. JAMA 2004;291:697-703.

29 Lew SQ, Sikka N: Are patients prepared to use telemedicine in home peritoneal dialysis programs? Perit Dial Int 2013;33:714-715.

30 Minatodani DE, Chao PJ, Berman SJ: Home telehealth: facilitators, barriers, and impact of nurse support among high-risk dialysis patients. Telemed J E Health 2013; 19:573.

31 Cao F, Li L, Lin M, Lin Q, Ruan Y, Hong F: Application of instant messaging software in the follow-up of patients using peritoneal dialysis, a randomised controlled trial. J Clin Nurs 2018;27: 3001-3007. 
32 Magnus M, Sikka N, Cherian T, Lew SQ: Satisfaction and improvements in peritoneal dialysis outcomes associated with telehealth. Appl Clin Inform 2017;08:214-225.

33 Jeffs L, Jain AK, Man RH, Onabajo N, Desveaux L, Shaw J, Hensel J, Agarwal P, Saragosa M, Jamieson T, Wong I, Maione M, Bhatia RS: Exploring the utility and scalability of a telehomecare intervention for patients with chronic kidney disease undergoing peritoneal dialysis-a study protocol. BMC Nephrol 2017;18:155.

34 Subramanian L, Tocco R, Fox, K, Sloand, James A, Perl J, Pisoni R, Kirk R, Perlman R, Adamowski T, Mccall, M, Perry E, Restovic K, Fitzpatrick L: Engaging Stakeholders in Protocol Development for Qualitative Research on Remote Management for Peritoneal Dialysis. American Society of Nephrology, Kidney Week - Abstract SA-PO694, 2017.

35 Cafazzo JA, Leonard K, Easty AC, Rossos PG, Chan CT: Patient perceptions of remote monitoring for nocturnal home hemodialysis. Hemodial Int 2010;14:471-477.

36 Liu N, Kim J, Jung Y, Arisy A, Nicdao MA, Mikaheal M, Baldacchino T, Khadra M, Sud K: Remote monitoring systems for chronic patients on home hemodialysis: field test of a copresence-enhanced design. JMIR Hum Factors 2017;4:e21.
37 Pierratos A: Nocturnal home haemodialysis: an update on a 5-year experience. Nephrol Dial Transplant 1999;14:2835-2840.

38 Tennankore KK, d'Gama C, Faratro R, Fung S, Wong E, Chan CT: Adverse technical events in home hemodialysis. Am J Kidney Dis 2015;65: 116-121.

39 Perl J, Davies SJ, Lambie M, Pisoni RL, McCullough K, Johnson DW, Sloand JA, Prichard S, Kawanishi H, Tentori F, Robinson BM: The peritoneal dialysis outcomes and practice patterns study (PDOPPS): unifying efforts to inform practice and improve global outcomes in peritoneal dialysis. Perit Dial Int 2016;36:297-307.

40 Manera KE, Johnson DW, Craig JC, Shen JI, Ruiz L, Wang AY, Yip T, Fung SKS, Tong M, Lee A, Cho Y, Viecelli AK, Sautenet B, Teixeira-Pinto A, Brown EA, Brunier G, Dong J, Dunning T, Mehrotra R, Naicker S, Pecoits-Filho R, Perl J, Wilkie $\mathrm{M}$, Tong A: Patient and caregiver priorities for outcomes in peritoneal dialysis: multinational nominal group technique study. Clin J Am Soc Nephrol 2019;14:74-83.

41 Jeffs L, Jain AK, Man RH, Onabajo N, Desveaux L, Shaw J, Hensel J, Agarwal P, Saragosa M, Jamieson T, Wong I, Maione M, Bhatia RS: Exploring the utility and scalability of a telehomecare intervention for patients with chronic kidney disease undergoing peritoneal dialysis - a study protocol. BMC Nephrol 2017;18:155.

Dr. Jeffrey Perl

Division of Nephrology, St. Michael's Hospital

30 Bond Street, 3-060 Shuter Wing

Toronto, ON M5B 1W8 (Canada)

E-Mail jeff.perl@utoronto.ca 